\title{
The decline of native Pacific island faunas: changes in status of the land snails of Samoa through the 20th century ${ }^{\text {is }}$
}

\author{
Robert H. Cowie ${ }^{\mathrm{a}, *}$, A.C. Robinson ${ }^{\mathrm{b}}$ \\ ${ }^{a}$ Center for Conservation Research and Training, University of Hawaii, 3050 Maile Way, Gilmore 408, Honolulu, HI 96822, USA \\ ${ }^{\mathrm{b}}$ Biodiversity Survey and Monitoring, National Parks and Wildlife, South Australia, Department for Environment and Heritage, \\ Box 1047, GPO, Adelaide, SA 5001, Australia
}

Received 6 March 2002; received in revised form 31 May 2002; accepted 1 June 2002

\begin{abstract}
The highly diverse and endemic Pacific island biota is disappearing and being replaced by a relatively small number of widespread alien species. The land snail fauna of Samoa (formerly Western Samoa) contains at least 72 species (58 native, 10 alien, four cryptogenic - of unknown origin). In 1992-1994 we surveyed the fauna in order to evaluate its status and, by comparison with previous surveys, to detect any trends. Twelve species have declined (eight native, two cryptogenic); 17 (15 native, two cryptogenic) show a "probable decline" or "possible decline"; five (four alien, one native) have increased or possibly increased. Some species showed no clear trend; others could not be evaluated, but some of them may be extinct. The fauna faces threats similar to those faced elsewhere, primarily habitat destruction and alien species impacts. Most notable is the introduction of a predatory flatworm, Platydemus manokwari, in attempts to control the giant African snail, Achatina fulica, which became established in Samoa in the 1990s. The flatworm may or may not be able to control $A$. fulica but poses a serious threat to the native snail fauna. Further introduction and distribution of alien predators should be strongly discouraged.
\end{abstract}

(C) 2002 Elsevier Science Ltd. All rights reserved.

Keywords: Alien species; Extinction; Gastropoda; Land snails; Pacific; Samoa

\section{Introduction}

Global biodiversity is declining at an alarming rate. The prime cause of this loss has long been recognized: habitat destruction, for urban and agricultural development and for natural resource exploitation. The impacts of alien species are now commonly regarded as second only to those of habitat destruction (e.g. Alonso et al., 2001; Simberloff, in press). Aliens may have direct impacts via competition, predation or herbivory, or indirect impacts through modification of native habitats and altering of community interactions (D'Antonio and Dudley, 1995; Simberloff, in press).

Endemic island biotas are particularly susceptible to losses caused by these two factors (Simberloff, 2000), which are combining on the islands of the tropical

\footnotetext{
t) Contribution number 2002-002 of Bishop Museum's Pacific Biological Survey.

* Corresponding author.

E-mail address: cowie@hawaii.edu (R.H. Cowie).
}

Pacific to cause a catastrophic loss of the islands' highly diverse and endemic plants and animals (Loope, 1998). In order to implement appropriate conservation management programs for this diversity (e.g. Sherley, 2000), the primary scientific requirements are knowledge of species distributions, identification of trends in these distributions, and understanding the causes of these trends. The great majority of Pacific island faunal diversity is composed of invertebrates (e.g. Eldredge, 2000), yet for many of these invertebrate groups little is known of either their past or present distributions. Without this knowledge it is impossible to develop adequate management strategies. With these needs in mind, this paper addresses the status of the highly endemic land snail fauna of Samoa.

Worldwide land snail diversity is second only to that of arthropods, and a large proportion of the world's land snail diversity is found on islands. Many islands, especially in the Pacific, harbor extremely diverse native land snail faunas (e.g. Solem, 1983; Cowie, 1996a, b). Few islands have been adequately surveyed (Cowie and 


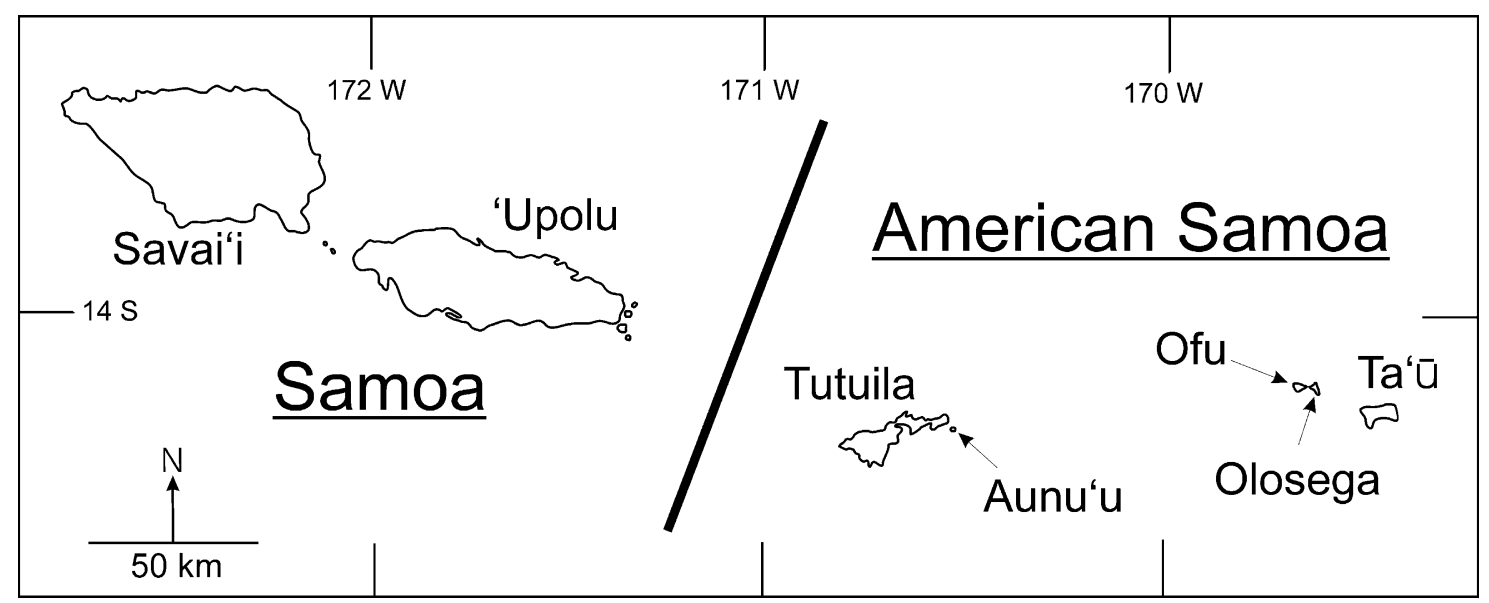

Fig. 1. The Samoan archipelago, excluding Rose Atoll and Swains Island.

Rundell, 2002; Cowie et al., in press). Nonetheless it is clear that many of these native Pacific island snail faunas have declined dramatically in the face primarily of habitat destruction, predation by introduced predators (particularly rats, predatory snails deliberately introduced in misguided attempts to control another introduced snail, the giant African snail, Achatina fulica, and possibly ants) and perhaps competition with introduced species (e.g. Hadfield, 1986; Kurozumi, 1988; Solem, 1990; Cowie, 1992, 2001a, b, 2002a; Hopper and Smith, 1992; Hadfield et al., 1993; Bauman, 1996; Bouchet and Abdou, 2001; Cowie and Cook, 2001). On many islands, the endemic snail species are largely confined to upper elevations and/or seem to be restricted to the remaining patches of primary forest. Overall, the native snail fauna of the Pacific islands is disappearing and being replaced by a small suite of widely distributed alien species (Cowie, 2002a).

Most of the species of native land snails in the Samoan archipelago were described during the latter half of the nineteenth century. During the twentieth century, survey work was undertaken in both American Samoa and Samoa (formerly Western Samoa) (for a brief historical summary see Cowie, 2001a), and some of the material collected was used in major taxonomic revisions of certain components of the Pacific land snail fauna (e.g. Baker, 1938, 1941; Cooke and Kondo, 1960; Solem, 1976, 1983). However, no faunal-wide assessment of the land snails of the Samoan islands had been undertaken until the recent nomenclatural catalog (Cowie, 1998), which lists all species, native and alien, recorded (up to 1998) in the Samoan islands, giving their island by island distributions. The results of recent survey work and an evaluation of the conservation status of the fauna of American Samoa have also recently been published (Cowie, 2001a; Cowie and Cook, 1999, 2001; Cowie and Rundell, 2002; Cowie et al., in press).

This paper reports the results of survey work carried out in 1992-1994 in [Western] Samoa, and, by comparing the results of this survey with those of earlier unpublished surveys, evaluates the conservation status of the native fauna of Samoa and the extent of its replacement by alien species. A similar approach has recently been adopted for assessing the status of Pacific island birds (Blanvillain et al., 2002). In combination with the recent evaluation of the American Samoan land snail fauna (references above), this paper concludes the most thorough, recent, fully published survey of the land snails of a large tropical Pacific archipelago.

\section{Study region}

The Samoan archipelago is a chain of volcanic islands extending in an approximately east-south-east to westnorth-west direction in the central southern Pacific (Fig. 1). It is generally considered a single biogeographic unit. Politically however, it comprises American Samoa (a territory of the United States) and Samoa (an independent state). This paper focuses on Samoa.

Samoa (Fig. 2) is composed of two main islands, Savai'i $\left(1718 \mathrm{~km}^{2}\right)$ and 'Upolu $\left(1125 \mathrm{~km}^{2}\right)$, a number of smaller islands, including Apolima $\left(5 \mathrm{~km}^{2}\right)$, Manono (10 $\left.\mathrm{km}^{2}\right)$, Nu'utele $\left(1.1 \mathrm{~km}^{2}\right)$, Nu'ulua $\left(0.3 \mathrm{~km}^{2}\right)$, and an additional 14 smaller islets. Savai' $i$ (elevation $1858 \mathrm{~m}$ ) is one of the highest and largest islands in Polynesia. 'Upolu $(1143 \mathrm{~m})$ is the second highest island in the group. All the smaller islands are considerably lower.

The Samoan islands are generally thought to have been formed as the Pacific plate moves across a stationary underlying "hot-spot", with magma periodically breaking through the crust to form the islands in chronological sequence, with the oldest (Savai ${ }^{i} i$ ) in the west and the youngest (the Manu'a islands, American Samoa) in the east (Keating, 1992). However, their ages are not well understood, in part because of active vulcanism on Savai'i that is probably associated with the proximity of the Tonga trench subduction zone, but 


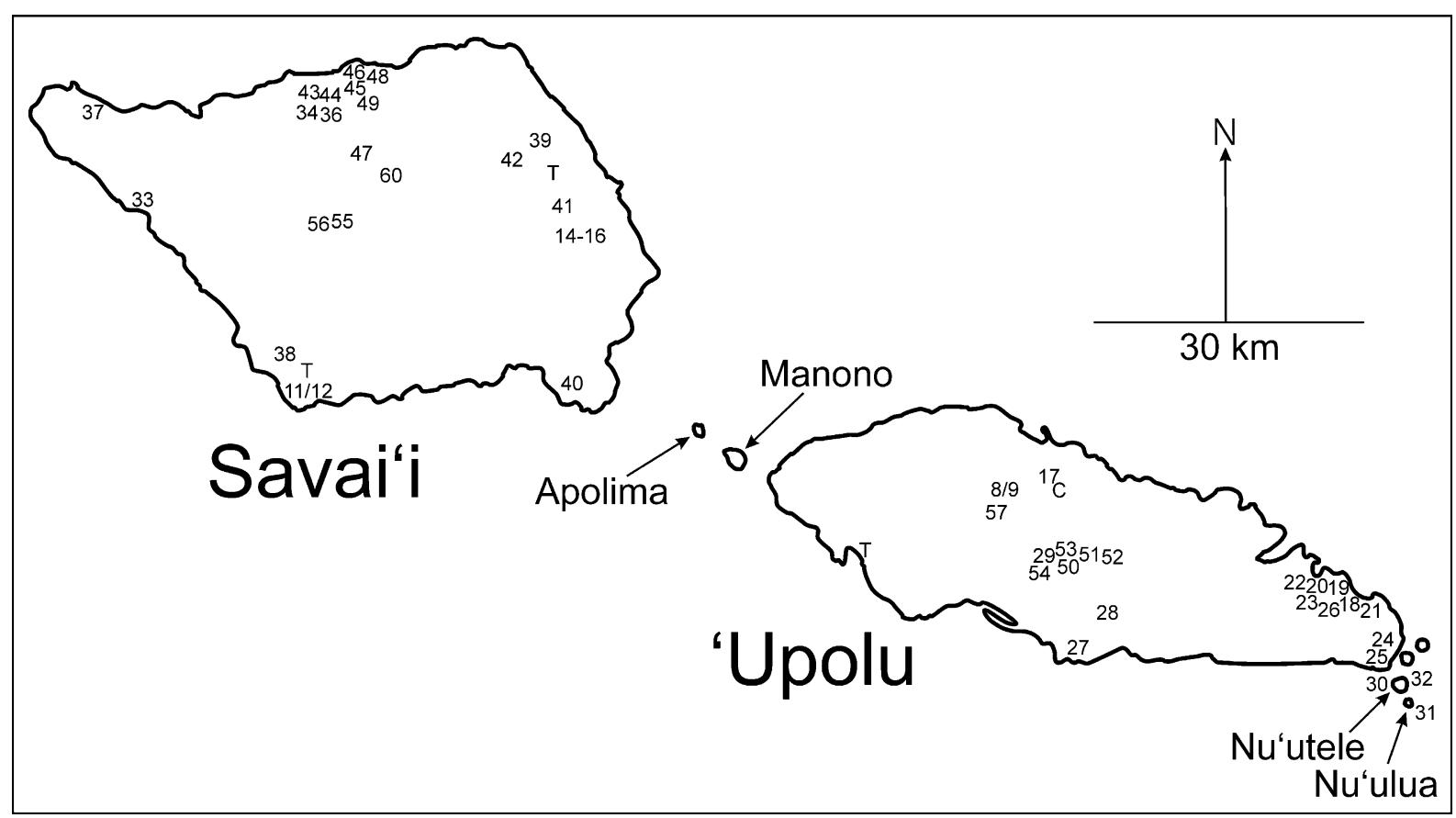

Fig. 2. Samoa, showing locations of 1992-1994 sampling locations. (ACR stations indicated by his field numbers; numbers 1-7, 10, 13, 35, 58, 59 are not part of this survey; numbers 8 and 9 are a single station, as are numbers 11 and 12, and 14-16. RHC station indicated by C; Pepper W. Trail stations indicated by $\mathrm{T}$.)

they appear to range between less than 1 Ma to about 4 Ma (Keating, 1992).

The islands were settled by Polynesians around 3000 years ago and the agricultural and hunting practices of these people undoubtedly had a dramatic effect on the indigenous biota, at least of the lowlands (e.g. Kirch, 1993; Hunt and Kirch, 1997), as they did elsewhere in Polynesia (Athens and Ward, 1993; Loope, 1998). However, modern urban development, extensive logging activity, and other habitat changes, mean that the islands now support much reduced and fragmented areas of natural habitat. Large numbers of alien plants and animals are now present, some resulting from early Polynesian introductions but many more from the ongoing introductions that followed discovery of the islands by Europeans. Further details of the Samoan environment have been provided by Taule'alo (1993), and the remaining relatively natural ecosystems have been described by Park et al. (1992), Pearsall and Whistler (1991a) and Whistler (1992, 1993, and references therein), and mapped by Pearsall and Whistler (1991b).

\section{Methods}

\subsection{Survey stations, sampling, identification}

Surveys were undertaken in 1992-1994 with the primary purpose of generating species inventories at each station as a means of evaluating overall species distributions. All sites identified as "grade 1" by Park et al. (1992), that is, good quality, relatively undisturbed, lowland rainforest, were sampled formally, as were a number of additional lowland and upland (above $450 \mathrm{~m}$ elevation) stations, resulting in 14 lowland and four upland stations on Savai'i, eight lowland and four upland stations on

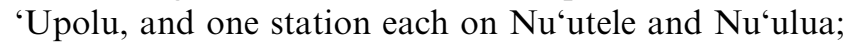
a total of 32 formally sampled stations. In other parts of the islands, snails were collected opportunistically, at an additional five stations on Savai'i, 10 on 'Upolu, and one on Nu'utele. Thus, an overall total of 48 stations was sampled, 23 on Savai'i, 22 on 'Upolu, two on $\mathrm{Nu}$ 'utele and one on $\mathrm{Nu}$ 'ulua (Fig. 2).

At each formally sampled station, sampling took place at intervals along a transect line and was by hand collecting of specimens in the field, both from vegetation and from the litter. This method probably undersamples smaller species (Ward-Booth and Dussart, 2001). However, it is the most efficient method for rapid inventory surveying as it yields many more specimens and many more species per unit of person-time than more intensive methods such as collecting litter for sorting in the laboratory (Emberton et al., 1996). It was similar to the approach adopted by Cowie (2001a), Cowie and Rundell (2002) and Cowie et al. (in press) in American Samoa. Almost all Samoan species are $<10$ $\mathrm{mm}$ in size, so any size-related sampling bias may not be great. Each station was searched for a period of at least $30 \mathrm{~min}$ by one or two people. Collections of up to five individuals of each species were made (except Partulidae and other rare endemics, which are readily identifiable 
in the field and for which only single specimens were usually taken).

All material was deposited in the Bishop Museum (Honolulu) malacological collections (accession number 1996.263, catalogue numbers BPBM 251260, 251261, collected by A.C. Robinson; 1996.278, 263537-263546, P.W. Trail; 2001.101, 263259-263534, 263710, A.C. Robinson and collaborators; 2001.091, 263248-263256, R.H. Cowie). Identification was by reference to previously identified material in the Bishop Museum. The collections have been databased and a basic subset of the data for each lot is available on the Samoan Snail Project website (www2.bishopmuseum.org/PBS/samoasnail/).

\subsection{Estimation of trends}

There has been no previous assessment of the conservation status of the land snail fauna of [Western] Samoa. However, quantitative estimates of extent of distribution and abundance at the times of earlier surveys (pre-1965 but mostly 1920-1940) were obtained by querying the database of identified specimens in the collections of the Bishop Museum, where almost all the pre-1965 twentieth century survey material is held. The definition of rarity is difficult as it combines the concepts of abundance, which may be very localized, and commonness, which may mean being widespread but sporadically distributed and not necessarily occurring in large numbers locally (Cameron, 1998). Using the same approach as did Cowie (2001a) for American Samoa, we attempted to distinguish between these concepts, as follows. With regard to extent of distribution, if there were collection lots (a "lot" being a collection of one species made at one place at one time) from more than one island (taking into account both Samoa and American Samoa) or if the lots came from a wide range of localities on the island to which a particular species was endemic, that species was considered "widespread". If the collection lots came from only one island and from only a few localities, that species was considered "highly localized". Regarding abundance, if there were 500 or more lots, that species was considered "abundant"; 100 or more but fewer than 500 , "common"; 50 or more but fewer than 100, "uncommon"; 10 or more but fewer than 50, "rare"; fewer than 10, "very rare". Necessarily, these assessments reflect the preferences of the original collectors in terms of collection localities, habitats, and species, but they are the best available data. For some species, there were no records in the database, suggesting that they were very rare even in the early twentieth century. The assessments were augmented by information from the primarily taxonomic literature dealing with the Achatinellidae (Cooke and Kondo, 1960), Pupillidae (Pilsbry, 1916-1918; Kirch, 1993), Endodontidae (Solem, 1976), Charopidae (Solem, 1983), and Helicarionidae (Baker, 1938, 1941), and from the very small amount of pre-1965 material in the Field Museum.

In 1965, Laurie Price and Alan Solem collected on Savai' $i$ and 'Upolu. They surveyed 40 stations: 28 on 'Upolu (station numbers 1-26, 39, 40) and 12 on Savai'i (27-38). Their collections are in the Field Museum of Natural History (Chicago). Data for the worldwide pulmonate land snail collections of the Field Museum have been databased and are available on line (www.fmnh.org/research_collections/). Data for the Price/Solem Samoan pulmonate collections were therefore obtained from this on line database. Data for Ostodes (Poteriidae), not yet on line, were obtained from the revision of Girardi (1978), which was based on the Price/Solem material. Data for other operculate land snails and for Ellobiidae, also not yet on line, were obtained from the Field Museum collections. For each species, we recorded the number of stations at which it was found on each island. Locations of survey stations 1-38 are in Solem (1983, p. 291), station 39 in Girardi (1978, p. 217) and Solem (1983, p. 201), and station 40 in Girardi (1978, p. 227). Station information for Endodontidae is also in Solem (1976), Charopidae in Solem (1983), and Ostodes in Girardi (1978).

In 1967, Yoshio Kondo collected on Savai'i and 'Upolu (Cowie, 2001a). His survey was not a faunalwide survey but focused almost exclusively on Partulidae. It is therefore not comparable with our 1992-1994 survey or with the historic surveys described above, and has not been used in our analysis.

Following the approach of Cowie (2001a), by qualitatively comparing the pre-1965 assessments of distribution and abundance and the data (number of stations at which each species was found) from the 1965 and 1992-1994 surveys, trends were assessed that reflect changes over almost the entire twentieth century. Species listed by Cowie (1998) but not included in these assessments were: those only known from American Samoa; those recorded only from "Samoa" (i.e. Samoa and/or American Samoa) with no island(s) specified; those recorded only questionably from [Western] Samoa; those that could not be identified definitively. Infraspecific names listed by Cowie (1998) were ignored.

\section{Results}

\subsection{Composition of the fauna}

Table 1 lists the species recorded during the 19921994 survey. It also provides the island distributions of species collected prior to the 1992-1994 survey, including those reported in the literature and hence listed by Cowie (1998), and those not previously reported in the literature but present in the Bishop Museum and/or Field Museum collections prior to 1992. It also lists the 
Table 1

Land snail and slug species of Samoa (assessment of trends based on pre-1965 estimates of distribution and abundance, the 1965 survey of Price and Solem, and the 1992-1994 survey reported here)

\begin{tabular}{|c|c|c|c|c|c|c|}
\hline Family and species ${ }^{\mathrm{a}}$ & $\begin{array}{l}\text { Islands known } \\
\text { from prior to the } \\
\text { 1992-1994 survey }\end{array}$ & Status pre- $1965^{\mathrm{c}}$ & $\begin{array}{l}\text { Island(s) and number } \\
\text { of stations in } 1965^{\mathrm{d}}\end{array}$ & $\begin{array}{l}\text { Island(s) and number } \\
\text { of stations in 1992-1994e }\end{array}$ & Trend & Origin $^{f}$ \\
\hline \multicolumn{7}{|l|}{ Helicinidae } \\
\hline Orobophana musiva (Gould, 1847) & S U & Widespread/common & $\mathrm{S} 2, \mathrm{U} 8$ & $\mathrm{~S} 1, \mathrm{U} 1$ & Decline & Indigenous \\
\hline Pleuropoma sp. ${ }^{\mathrm{g}}$ & S U & Widespread/common & $\mathrm{S} 1, \mathrm{U} 4$ & $\mathrm{~S} 3, \mathrm{U} 2$ & - & Endemic/indigenous \\
\hline Pleuropoma altivaga (Ancey, 1889) & $\mathrm{U}$ & Highly localized/very rare & $\mathrm{U} 2$ & $\mathrm{U} 1$ & Unknown & Endemic \\
\hline Pleuropoma beryllina (Gould, 1847) & $\mathrm{S}^{\dagger} \mathrm{U}$ & Widespread/abundant & $\mathrm{S}^{\dagger} 1, \mathrm{U} 2$ & $\mathrm{~S} 8, \mathrm{U} 8, \mathrm{Nt}^{\dagger} 2$ & Possible increase & Indigenous \\
\hline Pleuropoma jetschini (Wagner, 1905) & $\mathrm{U}$ & Not recorded & Not recorded & Not recorded & Unknown & Indigenous \\
\hline Pleuropoma fulgora (Gould, 1847) & S U & Widespread/abundant & S 9, U 26 & $\mathrm{~S} 15, \mathrm{U} 11, \mathrm{Nt}^{\dagger} 1, \mathrm{Nl}^{\dagger} 1$ & Possible decline & Indigenous \\
\hline Pleuropoma plicatilis (Mousson, 1865) & S U & Widespread/uncommon & $\mathrm{S} 11, \mathrm{U} 22$ & $\mathrm{~S} 3, \mathrm{U} 9$ & Possible decline & Endemic \\
\hline \multicolumn{7}{|l|}{ Neocyclotidae $^{i}$} \\
\hline Ostodes $\mathrm{sp}^{\mathrm{j}}$ & S U & Widespread/rare & - & S 14, U 6 & - & Endemic \\
\hline Ostodes cookei Clench, 1949 & U & Highly localized/very rare & Not recorded & Not recorded & Unknown & Endemic \\
\hline Ostodes exasperatus Girardi, 1978 & S U & Unknown & $\mathrm{S} 1, \mathrm{U} 1$ & Not recorded & Unknown & Endemic \\
\hline Ostodes garetti Clench, 1949 & $\mathrm{~S}$ & Highly localized/very rare & S 1 & Not recorded & Unknown & Endemic \\
\hline Ostodes gassiesi (Souverbie, 1859) & $\mathrm{S} \mathrm{U}$ & Unknown & S 5, U 17 & Not recorded & Possible decline & Endemic \\
\hline Ostodes llanero Girardi, 1978 & $\mathrm{~s}$ & Unknown & $\mathrm{S}_{2}$ & Not recorded & Unknown & Endemic \\
\hline Ostodes plicatus (Gould, 1847) & $\mathrm{S}^{\dagger} \mathrm{U}$ & Widespread/uncommon & $\mathrm{U} 6$ & U 4 & No clear trend & Endemic \\
\hline Ostodes reticulatus Girardi, 1978 & $\mathrm{U}$ & Unknown & U 9 & Not recorded & Possible decline & Endemic \\
\hline Ostodes savaii Clench, 1949 & $\mathrm{~S} \mathrm{U}$ & Widespread/rare & S $6, \mathrm{U} 13$ & Not recorded & Decline & Endemic \\
\hline Ostodes tiara (Gould, 1847) & $\mathrm{U}$ & Widespread/very rare & U 7 & $\mathrm{~S}^{\dagger} 2, \mathrm{U} 5$ & No clear trend & Endemic \\
\hline Ostodes upolensis (Mousson, 1865) & S U & Widespread/rare & $\mathrm{S} 1, \mathrm{U} 8$ & U 1 & Possible decline & Endemic \\
\hline \multicolumn{7}{|l|}{ Diplommatinidae } \\
\hline Diplommatina problematica (Mousson, 1865) & $\mathrm{S}^{\dagger} \mathrm{U}$ & Widespread/very rare & Not recorded & Not recorded & Possible decline & Endemic \\
\hline \multicolumn{7}{|l|}{ Truncatellidae } \\
\hline Truncatella guerinii Villa \& Villa, 1841 & $\mathrm{~S} \mathrm{U}^{\dagger}$ & Widespread/rare & $\mathrm{U} 1$ & $\mathrm{Nl}^{\dagger} 1$ & Unknown & Indigenous \\
\hline \multicolumn{7}{|l|}{ Assimineidae } \\
\hline Assiminea crosseana (Gassies, 1869) & $\mathrm{U}$ & Not recorded & Not recorded & Not recorded & Unknown & Indigenous \\
\hline Assiminea parvula (Mousson, 1865$)^{\mathrm{k}}$ & $\mathrm{S}^{\dagger} \mathrm{U}$ & Widespread/abundant & U 2 & Not recorded & Decline & Indigenous \\
\hline Assiminea similis (Baird, 1873) & $\mathrm{U}$ & Not recorded & Not recorded & Not recorded & Unknown & Indigenous \\
\hline Omphalotropis sp. 1 & $\mathrm{~S} \mathrm{U}$ & Widespread/common & S 8, U 3 & S 9, U 2 & & Endemic/indigenous \\
\hline Omphalotropis bifilaris Mousson, 1865 & $\mathrm{~S}^{\dagger} \mathrm{U}$ & Highly localized/very rare & $\mathrm{S}^{\dagger} 1, \mathrm{U} 1$ & Not recorded & Unknown & Endemic/indigenous \\
\hline Omphalotropis biliratus Mousson, 1865 & S U & Highly localized/very rare & $\mathrm{U} 1$ & Not recorded & Unknown & Endemic/indigenous \\
\hline Omphalotropis conoideus Mousson, 1865 & S U & Highly localized/very rare & $\mathrm{S} 7, \mathrm{U} 1$ & $\mathrm{~S} 9, \mathrm{U} 1$ & No clear trend & Endemic/indigenous \\
\hline \multicolumn{7}{|l|}{ Veronicellidae } \\
\hline Laevicaulis alte (Férussac, 1822) & $\mathrm{U}$ & Not recorded & U 1 & Not recorded & Unknown & Alien \\
\hline Vaginulus plebeius Fischer, 1868 & $\mathrm{U}$ & Highly localized/very rare & Not recorded & $\mathrm{S}^{\dagger} 2$ & Increase & Alien \\
\hline Unidentified Veronicellidae & - & Not recorded & Not recorded & $\mathrm{S}_{1}$ & - & Alien \\
\hline \multicolumn{7}{|l|}{ Ellobiidae } \\
\hline Auriculastra subula (Quoy \& Gaimard, 1832) & $\mathrm{U}$ & Highly localized/very rare & Not recorded & Not recorded & Unknown & Indigenous \\
\hline Ellobium semisculptum Adams \& Adams, 1854 & $\mathrm{U}$ & Not recorded & Not recorded & Not recorded & Unknown & Indigenous \\
\hline Melampus spp. $^{\mathrm{m}}$ & $\mathrm{S} \mathrm{U}$ & Widespread/common & $\mathrm{U} 1$ & $\mathrm{U} 2, \mathrm{Nt}^{\dagger} 1, \mathrm{Nl}^{\dagger} 1$ & Unknown & Indigenous \\
\hline Allochroa layardi (Adams \& Adams, 1855) & $\mathrm{U}$ & Highly localized/very rare & Not recorded & Not recorded & Unknown & Indigenous \\
\hline Cassidula sp." & $\mathrm{U}$ & Highly localized/very rare & Not recorded & Not recorded & - & Indigenous \\
\hline Cassidula crassiuscula Mousson, 1869 & $\mathrm{U}$ & Not recorded & Not recorded & Not recorded & Unknown & Indigenous \\
\hline Cassidula paludosa (Garrett, 1872) & $\mathrm{U}$ & Not recorded & Not recorded & Not recorded & Unknown & Indigenous \\
\hline Pythia sp. ${ }^{\circ}$ & $\mathrm{Nt}^{\dagger}$ & Highly localized/very rare & Not recorded & - & & Indigenous \\
\hline Pythia savaiensis Mousson, $1869^{\circ}$ & $\mathrm{S}$ & Not recorded & Not recorded & Not recorded & Unknown & Indigenous \\
\hline Pythia scarabaeus (Linnaeus, 1868) & - & Widespread/rare & Not recorded & $\mathrm{S}^{\dagger} 2, \mathrm{Nt}^{\dagger} 1, \mathrm{Nl}^{\dagger} 1$ & Probably no change & Indigenous \\
\hline Pythia tortuosa Mousson, $1871^{\circ}$ & $\mathrm{U} \mathrm{S}^{\dagger}$ & Highly localized/very rare & Not recorded & Not recorded & Unknown & Indigenous \\
\hline
\end{tabular}




\begin{tabular}{|c|c|c|c|c|c|c|}
\hline Family and species ${ }^{\mathrm{a}}$ & $\begin{array}{l}\text { Islands known } \\
\text { from prior to the } \\
\text { 1992-1994 survey }\end{array}$ & Status pre- $1965^{\mathrm{c}}$ & $\begin{array}{l}\text { Island(s) and number } \\
\text { of stations in } 1965^{\mathrm{d}}\end{array}$ & $\begin{array}{l}\text { Island(s) and number } \\
\text { of stations in 1992-1994e }\end{array}$ & Trend & Origin $^{f}$ \\
\hline $\begin{array}{l}\text { Achatinellidae } \\
\text { Lamellidea } \mathrm{sp}(\mathrm{p}){ }^{\mathrm{p}} \\
\text { Elasmias } \mathrm{sp} .\end{array}$ & $\begin{array}{l}\mathrm{U} \\
\mathrm{U}\end{array}$ & $\begin{array}{l}\text { Widespread/uncommon } \\
\text { Widespread/rare }\end{array}$ & $\begin{array}{l}1 \\
<10\end{array}$ & $\begin{array}{l}\text { Not recorded } \\
\text { Not recorded }\end{array}$ & $\begin{array}{l}\text { Possible decline } \\
\text { Possible decline }\end{array}$ & $\begin{array}{l}\text { Cryptogenic } \\
\text { Cryptogenic }\end{array}$ \\
\hline $\begin{array}{l}\text { Pupillidae } \\
\text { Gastrocopta pediculus } \text { (Shuttleworth, 1852) } \\
\text { Nesopupa godeffroyi }(\text { Boettger, 1881) } \\
\text { Pupisoma orcula }(\text { Benson, } 1850)^{\mathrm{r}}\end{array}$ & $\begin{array}{l}\mathrm{U} \\
\mathrm{S}^{\dagger} \mathrm{U}^{\dagger} \\
\mathrm{U}^{\dagger}\end{array}$ & $\begin{array}{l}\text { Widespread/uncommon } \\
\text { Widespread/uncommon } \\
\text { Widespread/rare }\end{array}$ & $\begin{array}{l}\text { Not recorded } \\
\text { U } 1 \\
\text { Not recorded }\end{array}$ & $\begin{array}{l}\text { Not recorded } \\
\text { Not recorded } \\
\text { Not recorded }\end{array}$ & $\begin{array}{l}\text { Decline } \\
\text { Decline } \\
\text { Decline }\end{array}$ & $\begin{array}{l}\text { Cryptogenic } \\
\text { Endemic } \\
\text { Cryptogenic }\end{array}$ \\
\hline $\begin{array}{l}\text { Partulidae } \\
\text { Eua expansa (Pease, 1871) } \\
\text { Eua montana (Cooke \& Crampton, 1930) } \\
\text { Samoana canalis (Mousson, 1865) } \\
\text { Samoana stevensoniana (Pilsbry, 1909) }\end{array}$ & $\begin{array}{l}\text { S U } \\
\text { U } \\
\text { S U } \\
\text { S U }\end{array}$ & $\begin{array}{l}\text { Widespread/uncommon } \\
\text { Highly localized/very rare } \\
\text { Widespread/uncommon } \\
\text { Widespread/rare }\end{array}$ & $\begin{array}{l}\text { S } 7, \text { U } 10 \\
\text { U } 3 \\
\text { S } 9, \text { U } 7 \\
\text { S } 10, \text { U } 3\end{array}$ & $\begin{array}{l}\text { S } 10, \text { U } 2 \\
\text { U } 4 \\
\text { S } 5, \text { U } 2 \\
\text { S } 2\end{array}$ & $\begin{array}{l}\text { Possible decline } \\
\text { Unknown } \\
\text { Possible decline } \\
\text { Decline }\end{array}$ & $\begin{array}{l}\text { Endemic } \\
\text { Endemic } \\
\text { Endemic } \\
\text { Endemic }\end{array}$ \\
\hline $\begin{array}{l}\text { Subulinidae } \\
\text { Allopeas gracile (Hutton, 1834) } \\
\text { Opeas hannense (Rang, 1831) } \\
\text { Paropeas achatinaceum (Pfeiffer, 1846) } \\
\text { Subulina octona (Bruguière, 1789) }\end{array}$ & $\begin{array}{l}\mathrm{S}^{\dagger} \mathrm{U} \\
\mathrm{U} \\
\mathrm{S}^{\dagger} \mathrm{U}^{\dagger} \\
\mathrm{S}^{\dagger} \mathrm{U}^{\dagger}\end{array}$ & $\begin{array}{l}\text { Widespread/common } \\
\text { Widespread/common } \\
\text { Widespread/abundant } \\
\text { Widespread/abundant }\end{array}$ & $\begin{array}{l}\mathrm{U}<3 \\
\mathrm{U} 2 \\
\mathrm{~S} 11, \mathrm{U} 20 \\
\mathrm{~S} 7, \mathrm{U} 13\end{array}$ & $\begin{array}{l}\mathrm{Nl}^{\dagger} 1 \\
\text { Not recorded } \\
\text { S } 16, \mathrm{U} 15, \mathrm{Nt}^{\dagger} 1 \\
\text { S } 12, \mathrm{U} 12, \mathrm{Nt}^{\dagger} 2, \mathrm{Nl}^{\dagger} 1\end{array}$ & $\begin{array}{l}\text { Decline } \\
\text { Decline } \\
\text { No clear trend } \\
\text { No clear trend }\end{array}$ & $\begin{array}{l}\text { Polynesian introd. } \\
\text { Alien } \\
\text { Alien } \\
\text { Alien }\end{array}$ \\
\hline $\begin{array}{l}\text { Achatinidae } \\
\text { Achatina fulica Bowdich, } 1822\end{array}$ & $\mathrm{U}$ & Not recorded & Not recorded & Not recorded & Increase & Alien \\
\hline $\begin{array}{l}\text { Rhytididae } \\
\text { Ouagapia gradata (Gould, 1846) }\end{array}$ & $\mathrm{S} \mathrm{U}$ & Widespread/common & S 1, U 11 & Not recorded & Decline & Indigenous \\
\hline $\begin{array}{l}\text { Plectopylididae } \\
\text { Corilla carabinata (Férussac, 1821) }\end{array}$ & $\mathrm{U}$ & Highly localized/very rare & Not recorded & Not recorded & Unknown & Alien \\
\hline $\begin{array}{l}\text { Endodontidae } \\
\text { Thaumatodon hystricelloides (Mousson, 1865) }\end{array}$ & $\mathrm{U}$ & Widespread/common & U 3 & Not recorded & Decline & Endemic \\
\hline $\begin{array}{l}\text { Charopidae }^{\mathrm{v}} \\
\text { Graeffedon graeffei }(\text { Mousson, 1869) } \text { Graeffedon savaiiensis Solem, 1983 } \\
\text { Sinployea allecta } \text { (Cox, 1870) } \\
\text { Sinployea clista Solem, 1983 } \\
\text { Sinployea complementaria (Mousson, 1865) }\end{array}$ & $\begin{array}{l}\mathrm{U} \\
\mathrm{S} \\
\mathrm{S} \mathrm{U} \\
\mathrm{U} \\
\mathrm{U}\end{array}$ & $\begin{array}{l}\text { Highly localized/very rare } \\
\text { Highly localized/very rare } \\
\text { Widespread/very rare } \\
\text { Widespread/very rare } \\
\text { Unknown/very rare }\end{array}$ & $\begin{array}{l}\text { U } 1 \\
\text { Not recorded } \\
\text { S 2, U } 3 \\
\text { U } 2 \\
\text { U } 8\end{array}$ & $\begin{array}{l}\text { Not recorded } \\
\text { Not recorded } \\
\text { Not recorded } \\
\text { Not recorded } \\
\text { Not recorded }\end{array}$ & $\begin{array}{l}\text { Unknown } \\
\text { Unknown } \\
\text { Possible decline } \\
\text { Possible decline } \\
\text { Possible decline }\end{array}$ & $\begin{array}{l}\text { Endemic } \\
\text { Endemic } \\
\text { Endemic } \\
\text { Endemic } \\
\text { Endemic }\end{array}$ \\
\hline $\begin{array}{l}\text { Succineidae } \\
\text { Succinea crocata Gould, } 1846 \\
\text { Succinea modesta Gould, } 1846^{\mathrm{w}} \\
\text { Succinea putamen Gould, } 1846\end{array}$ & $\begin{array}{l}\mathrm{U} \\
\mathrm{U} \\
\mathrm{U}\end{array}$ & $\begin{array}{l}\text { Widespread/rare } \\
\text { Widespread/uncommon } \\
\text { Widespread/rare }\end{array}$ & $\begin{array}{l}\text { U } 1 \\
\text { Not recorded } \\
\text { U } 2\end{array}$ & $\begin{array}{l}\text { U } 2 \\
S^{\dagger} 5, \mathrm{U} 1 \\
\mathrm{~S}^{\dagger} 4, \mathrm{U} 7\end{array}$ & $\begin{array}{l}\text { Possible decline } \\
\text { No clear trend } \\
\text { No clear trend }\end{array}$ & $\begin{array}{l}\text { Endemic } \\
\text { Endemic } \\
\text { Endemic }\end{array}$ \\
\hline $\begin{array}{l}\text { Helicarionidae }^{\mathrm{x}} \\
\text { Diastole sp. } \\
\text { Diastole lamellaxis Baker, } 1938 \\
\text { Diastole savaii } \text { Baker, } 1938 \\
\text { Diastole schmeltziana (Mousson, 1865) } \\
\text { Lamprocystis perpolita (Mousson, 1869) } \\
\text { Lamprocystis upolensis (Mousson, 1865) } \\
\text { Liardetia samoensis (Mousson, 1865) }\end{array}$ & $\begin{array}{l}- \\
\text { S } \\
\text { S } \\
\text { S U } \\
\text { S U } \\
\text { S U } \\
S^{\dagger} U\end{array}$ & $\begin{array}{l}\text { Highly localized/rare } \\
\text { Highly localized/rare } \\
\text { Widespread/common } \\
\text { Highly localized/very rare } \\
\text { Widespread/very rare } \\
\text { Widespread/common }\end{array}$ & $\begin{array}{l}- \\
\text { Not recorded } \\
\text { S } 1 \\
\text { S } 8, \text { U } 20 \\
\text { S } 1, \text { U } 1 \\
\text { Not recorded } \\
\text { S }^{\dagger} 1, \mathrm{U} 4\end{array}$ & $\begin{array}{l}\text { S 2, U } 2 \\
\text { S } 1 \\
\text { Not recorded } \\
\text { U } 1 \\
\text { Not recorded } \\
\text { Not recorded } \\
\text { Not recorded }\end{array}$ & $\begin{array}{l}- \\
\text { Unknown } \\
\text { Unknown } \\
\text { Possible decline } \\
\text { Unknown } \\
\text { Possible decline } \\
\text { Decline }\end{array}$ & $\begin{array}{l}\text { Endemic/indigenous } \\
\text { Endemic } \\
\text { Endemic } \\
\text { Endemic } \\
\text { Endemic } \\
\text { Indigenous } \\
\text { Indigenous }\end{array}$ \\
\hline $\begin{array}{l}\text { Ariophantidae } \\
\text { Parmarion martensi Simroth, } 1893\end{array}$ & $\mathrm{U}$ & Not recorded & Not recorded & U 4 & Increase & Alien \\
\hline $\begin{array}{l}\text { Zonitidae } \\
\text { Trochomorpha sp. }\end{array}$ & - & - & - & $\mathrm{U} 1, \mathrm{Nt}^{\dagger} 1$ & - & Endemic \\
\hline
\end{tabular}


Table 1 (continued)

\begin{tabular}{|c|c|c|c|c|c|c|}
\hline Family and species ${ }^{\mathrm{a}}$ & $\begin{array}{l}\text { Islands known } \\
\text { from prior to the } \\
\text { 1992-1994 survey }\end{array}$ & Status pre- $1965^{\circ}$ & $\begin{array}{l}\text { Island(s) and number } \\
\text { of stations in } 1965^{\mathrm{d}}\end{array}$ & $\begin{array}{l}\text { Island(s) and number } \\
\text { of stations in 1992-1994 }\end{array}$ & Trend & Origin $^{\mathrm{f}}$ \\
\hline Trochomorpha apia (Hombron \& Jacquinot, 1852) & $\mathrm{S} \mathrm{U}$ & Widespread/common & U 1 & S $8, \mathrm{U} 2$ & Probable decline & Endemic \\
\hline Trochomorpha samoa (Hombron \& Jacquinot, 1841) & $\mathrm{U}$ & Not recorded & Not recorded & Not recorded & Unknown & Endemic \\
\hline Trochomorpha troilus (Gould, 1846) & S U & Widespread/rare & U 1 & $\mathrm{~S} 2, \mathrm{U} 6$ & No clear trend & Endemic \\
\hline Trochomorpha tuber Mousson, 1869 & $\mathrm{U}$ & Highly localized/very rare & Not recorded & Not recorded & Unknown & Endemic \\
\hline $\begin{array}{l}\text { Bradybaenidae } \\
\text { Bradybaena similaris (Rang, 1831) }\end{array}$ & $\mathrm{SU}$ & Widesnered/uncommon & $S 3=5$ & S $10 \mathrm{JU} 0$ & Poscible increase & Alien \\
\hline
\end{tabular}

${ }^{a}$ Taxonomic arrangement and nomenclature follow Cowie (1998).

b S, Savai i'; U, 'Upolu; Nt, Nu'utele; Nl, Nu'ulua. Islands from which the species were previously known, as given by Cowie (1998), and, indicated by a dagger, species not reported by Cowie (1998) but for which there are specimens in the Bishop Museum and/or Field Museum collected prior to the 1992-1994 survey (i.e. new published records for the island).

is given, this information is lost. Newly published island records indicated by a dagger $(\dagger)$.

Bio

Biogeographic origin from Cowie (19). Endemic, occurs only in the Samoan islands (Samoa, American Samoa); indigenous, occurs naturally in the Samoan islands and elsewhere; cryptogenic, unclear whether native or alien in

he Samoan islands; Polynesian introd, introduced prior to European discovery of the Samoan islands; alien, introduced subsequent to European discovery of the islands.

Tentatively identified. May be Pleuropoma jetschini or a mixture of $P$. beryllina, $P$. jetschini, and perhaps an undescribed species. However, may be one variable species and these may be synonyms (see Cowie, 2001a). Perhaps also the reason $P$. jetschini has never been recorded since its original description.

i Assessments based on Clench (1949) and Girardi (1978) as well as the Bishop Museum database.

j Ostodes species are highly variable and difficult to identify, with some species not described until 1949 and 1978 . Hence, much early material as well as some of the present material has only been referred to Ostodes sp. The recor of Ostodes strigatus from 'Upolu in the Bishop Museum database (catalog number BPBM 193) is considered in error, following Clench (1949), Girardi (1978) and Cowie (1998).

${ }_{\mathrm{k}}$ Assiminea crosseana and $A$. similis may be synonyms of $A$. parvula [the commonly used Assiminea nitida (Pease, 1865) is also a synonym]; alternatively, some specimens may have been incorrectly referred to $A$. parvula. The recor of A. parvula from Savai'i is based on a single collection lot (BPBM 108317) referred to Assiminea sp. in the Bishop Museum database. The pre-1965 assessments of A. parvula are based largely on lots referred to Assiminea sp. in the database.

The taxonomy of Samoan Omphalotropis is in need of revision and the species are difficult to identify. Very few specimens in the Bishop Museum have been identified to species, which probably explains the "highly localized/very rare" assessment for the three named species. Most of the Price/Solem 1965 material was only tentatively referred to Omphalotropis conoideus. The Price/Solem record of $O$. bifilaris fom Savai'i is of the subspecies teretiform Mousson, 1869. Determining these species' status as endemic or indigenous depends on future revision.

$\mathrm{m}$ Because the various species of Melampus are difficult to distinguish and much of the material in the Bishop Museum collections is identified only as Melampus sp., all records of species of Melampus are combined (cf. Cowie, 2001a). They include: Melampus castaneus (Megerle von Mühlfeld, 1816) (reported in [Western] Samoa from 'Upolu; no specimens in the Bishop Museum database), Melampus fasciatus (Deshayes, 1830) (Savai'i and 'Upolu; one pre1965 specimen lot only, from American Samoa), Melampus luteus (Quoy \& Gaimard, 1832) ('Upolu; five pre-1965 lots, from American Samoa), Melampus philippii (Küster, 1845) ('Upolu; one lot, from American Samoa), Melamp semisulcatus Mousson, 1869 (Savai' and 'Upolu, no specinens), Melampus siniums Pease, 1861 (Upolu, no specimens).

n Probably referable to either Cassidula crassiuscula or C. paludosa, which may be synonyms.

Probably Pythia scarabaeus.

p Records of Lamellidea oblonga (Pease, 1865) and Lamellidea pusilla (Gould, 1847), both recorded from American Samoa and widespread in the Pacific, have been combined. They may not be distinct species and many records q be misidentifications.

q Assessments based on Bishop Museum database records of Gastrocopta sp. and G. pediculus combined.

${ }^{\mathrm{r}}$ Assessments based on Bishop Museum database records of Nesopupa sp. and N. godeffroyi combined, and of the single Field Museum database record of Nesopupa sp.

${ }^{t}$ The few records of Samoana conica (Gould, 1847) from 'Upolu, which is otherwise known only from American Samoa, may be misidentifications of S. canalis, or the two may be synonyms (see Cowie, 1998). S. conica is omitted from this treatment.

" Assessment based on Solem (1983, p. 455), referring to the 1860s and 1870s. An assessment based only on Bishop Museum collections would be "highly localized/very rare".

v Assessments based on Solem (1983) as well as the Bishop Museum database.

Those from the main islands (i.e. excluding the Manu'a islands) are considered mostly to be referable to $S$. modesta.

(1938) and this may be a misidentification. 
species collected by Price and Solem in 1965. From these combined sources, the total number of land snail (and slug) species recorded from Samoa is 72 (a slight under-counting because all Melampus spp. were counted as one species). Of these, 58 are native (35-38 endemic to the Samoan islands); one is a Polynesian, pre-European introduction; nine are more recent introductions; and four are cryptogenic (of unknown native or alien origin-Carlton, 1996).

Review of pre-1992 collections in the Bishop Museum (and the very small amount of pre-Price/Solem 1965 material in the Field Museum) resulted in 14 new island records for 11 species (including those listed as "sp." only if they represented a new island record for the genus); these new island records included new records for [Western] Samoa for four species:Pupisoma orcula (cryptogenic; also in American Samoa and recorded by Cowie, 2001a), Nesopupa godeffroyi (endemic to the archipelago; also in American Samoa), Paropeas achatinaceum (alien), Subulina octona (alien) (both these latter two species also in American Samoa, in great abundance, and recorded by Cowie, 2001a).

Records of much of the Price/Solem 1965 material have never been published. Their records of Pleuropoma beryllina, Omphalotropis bifilaris (as subspecies teretiformis) and Liardetia samoensis are the first records of these species for Savai'i.

The 1992-1994 survey recorded at least 29 species (all Melampus spp. combined as one). Of these, 23 were native and six were alien (including the single Polynesian introduction). A single species (Pythia scarabaeus) was recorded from [Western] Samoa for the first time, although previous records of other Pythia species are probably referable to this species. The known distributions of 13 species were increased (18 new island records).

\subsection{Trends}

Differences in locations and methods between our 1992-1994 survey and previous surveys preclude truly rigorous comparison. However, these data are the best available and some probably reliable overall trends can indeed be detected.

Our qualitative evaluations of the changes in distribution and/or abundance of the species during the twentieth century are presented in Table 1. Overall, 12 species appear to have declined. Of these, the majority (eight) are native species, and two are cryptogenic. An additional 17 species (15 native, two cryptogenic) show a "probable decline" or "possible decline". Five species (four alien, one native) appear to have increased or possibly increased. For species that appeared extremely rare even in the early twentieth century and that were collected in low numbers or not at all in the 1965 and 1992-1994 surveys a clear assessment is not possible and any change in distribution/abundance is stated as "unknown". Some species that were widespread and in some cases abundant early in the twentieth century, and remain so, show "no clear trend"; for some we can make a tentative assessment of "probably no decline" or "probably no change".

Some species remain relatively common on Savai'i but appear to have declined on 'Upolu (e.g. Omphalotropis conoideus, Trochomoprha apia). Some native species (e.g. Ostodes tiara, Succinea crocata, S. putamen, Trochomorpha troilus) also appear to remain more common at higher elevations, while some of the aliens are more common at lower elevations (e.g. Subulina octona, Laevicaulis alte).

\section{Discussion}

\subsection{The fauna}

The native land snail fauna of the Samoan islands as listed by Cowie (1998) with additional records by Cowie (2001a) and Cowie et al. (in press) included 99 native species. Of these, 64 are known from Samoa and 47 from American Samoa (some are common to both). Table 1 lists only 58 native species in Samoa because a number of nomenclaturally valid but unidentifiable species (two helicinids and an assimineid) and a possible misidentification (Samoana conica) have been omitted, the six nominal species of Melampus have been combined as "Melampus spp.", but three native species are recorded for the first time (Pleuropoma beryllina, Pythia scarabaeus, Nesopupa godeffroyi). The native fauna of Samoa is thus approximately 30\% more speciose than that of American Samoa, possibly because the total land area of Samoa is much greater and because the two large islands ('Upolu, Savai'i) are much larger than the single large island of American Samoa (Tutuila).

The non-native faunas also differ. The combined records of Cowie (1998, 2001a), Cowie and Rundell (2002), Cowie et al. (in press), and the present report, give totals of 20 alien species (plus seven cryptogenic species) in American Samoa but only 10 (plus four cryptogenic) in Samoa. Thus, not only do the numbers of species in Samoa and American Samoa differ but the proportions of native to alien (including cryptogenic) species are radically different. Of the total fauna of Samoa, 21\% (14 of 72) of the species are alien/cryptogenic, whereas in American Samoa this figure is $36 \%$ (27 of 74). This difference is statistically significant (loglikelihood $G$-test, $G=5.288,1$ d.f., $P=0.021)$. It may be a reflection of the much larger areas in Samoa that remain relatively unspoiled compared with American Samoa, which in turn reflects the human population pressure: the most recent figures available (Dahl, 1991) are 54 people per $\mathrm{km}^{2}$ in Samoa (1986 data), 172 per $\mathrm{km}^{2}$ in American Samoa (1982 data). 


\subsection{Status of the species and threats to them}

Fewer alien species have been recorded in Samoa than in American Samoa, but the trends are similar in both. Alien species appear to be increasing; native species are declining. In Samoa, as in American Samoa (Cowie, 2001a), these apparent trends seem convincing despite the limitations of the various surveys and the impossibility of drawing quantitative conclusions based on carefully replicated data.

For a number of species we could make no evaluation or could identify no clear trend. In many cases these were native species that had probably declined long ago (even before the early twentieth century surveys) and that may be extinct (e.g. Endodontidae, Charopidae) (see also Solem, 1976, 1983), or alien species that were introduced long ago and were already widespread and abundant by the early twentieth century (e.g. some of the Subulinidae).

Partulid tree snails in particular have achieved renown among Pacific island non-marine snails as being especially vulnerable (e.g. Gould, 1991); they have received more attention than any other group (e.g. Cowie, 1992; Johnson et al., 1993; Coote, et al., 1999; Goodacre and Wade, 2001); and have been termed the flagships of terrestrial invertebrate conservation in the Pacific (Cowie and Cook, 2001). Partulids are endemic to the islands of the Pacific, and most species occurr on only one island or within a single archipelago (Cowie, 1992). The four partulid species of Samoa could still be found in 1992-1994, as could the partulids of American Samoa in 1998 (Cowie and Cook, 2001). At least on Savai'i, Eua expansa remains fairly widespread, although it may have declined on 'Upolu. E. montana is an upper elevation species that has never been considered common. Samoana canalis remained in a number of localities, though it was not abundant and may be declining. S. stevensoniana has probably declined since 1965. Overall, the Samoan partulids are probably declining and are certainly threatened, as are partulids elsewhere (including in American Samoa).

Two other species deserve mention. The native helicinid Pleuropoma beryllina remains widespread and abundant, as it does in American Samoa, where it was the most numerous species collected in the recent survey (Cowie, 2001a). However, whereas in American Samoa the native helicarionid Diastole schmeltziana was considered stable or even increasing (Cowie, 2001a), it seems to have declined dramatically in Samoa, based on the 1965 to 1992-1994 comparisons (Table 1). Even if unidentified Diastole sp. records are assumed to represent D. schmeltziana, the trend remains. This contrast is unexplained.

The apparent scarcity of Ellobiidae and Truncatellidae in 1965 and 1992-1994 probably reflects undersampling of their supralittoral habitat, rather than real declines, although some of these species, while widespread, are naturally sparsely distributed.

No doubt the reasons underlying these changes in the fauna are similar in both American Samoa and Samoa (as well as elsewhere throughout the Pacific) and include: habitat modification, as a result of both invasion of alien plants and more direct destruction resulting from human development (agriculture, logging, urban expansion); possible competitive interactions between native and alien species, although this remains speculative; and predation by alien species. Cowie (2001a) discussed these threats more fully.

One of these threats, however, is of particular significance. Introduction of the giant African snail (Achatina fulica) to 'Upolu during the 1990s (not recorded in the 1992-1994 survey) poses perhaps the greatest immediate threat to the native Samoan fauna. Its introduction in 1977 to American Samoa (at present only on Tutuila and $\mathrm{Ta}^{\prime} \mathrm{u}$ ) led to the introduction of the predatory snail Euglandina rosea (and perhaps other predatory snail species) as an ill-considered putative biological control agent. The efficacy of E. rosea as a biocontrol agent has not been demonstrated (Cowie, 2001b), but it is now a serious threat to the native snail fauna. Elsewhere in the Pacific it has caused or been seriously implicated in the extinction of many native snail species (e.g. Murray et al., 1989; Hadfield, 1986; Cowie, 1992; Hopper and Smith, 1992; Hadfield et al., 1993; Civeyrel and Simberloff, 1996; Coote et al., 1999). As yet, it has not been introduced to Samoa. However, a perhaps even more serious snail predator, the flatworm Platydemus manokwari, has recently been introduced and is being reared in Samoa with the prospect of dispersing it widely as a control agent against $A$. fulica (Cowie, 2002b). P. manokwari has been seriously implicated in the decline of native snails elsewhere in the Pacific (Hopper and Smith, 1992).

\subsection{Conclusion}

The overall trend in Samoa is of decline of the native land snail fauna and its replacement by alien snail species, as is the case throughout the Pacific (Cowie, 2002a). However, this trend may be less dramatic in Samoa than in American Samoa. Furthermore, within Samoa, the fauna seems to be faring better on Savai'i than on 'Upolu, which probably reflects 'Upolu's smaller size, lower maximum elevation, and greater human population pressure. Nonetheless, there is no cause for complacency. The giant African snail (Achatina fulica) is now established on 'Upolu and efforts underway to control it could have serious consequences for the native snail fauna (see above). As yet, A. fulica is not on Savai' $i$, but should it get there, the temptation to introduce $P$. manokwari will be strong.

The native land snail faunas of Pacific islands exhibit high levels of endemism and diversity. However, they are 
perhaps the most vulnerable members of these island biotas (Paulay, 1994). Extinction rates are dramatic. For instance, of Hawaii's over 750 species (Cowie, 1996a), $75 \%$ (Solem, 1990) or as many as $90 \%$ (R.H. Cowie, unpublished) are extinct. In the Ogasawara Islands $40 \%$ of the 114 species have become extinct since the 1860s (Tomiyama and Kurozumi, 1992). On Rota (Northern Marianas) $68 \%$ of the 43 species are extinct or declining (Bauman, 1996). All the Partulidae of Moorea (French Polynesia) are extinct in the wild (Murray et al., 1989).

The native snail fauna of Samoa, although declining, does not seem to be quite so imminently endangered. These species therefore deserve special attention because we now have an opportunity to preserve an important component of the Pacific island biota and perhaps prevent these species from going the way of many of the other unique Pacific island land snails. In particular, efforts must be made to discourage the further introduction and spread of alien predators.

\section{Acknowledgements}

RHC thanks the US National Science Foundation (grant DEB 9705494), the US National Park Service, the US Fish and Wildlife Service, and the US Geological Survey (Biological Resources Division) for support of his work in the Samoan islands. ACR spent 2 years in Samoa working for the Department of Environment and Conservation as part of the Australian Volunteers Abroad Scheme administered by the Overseas Service Bureau; he thanks the South Pacific Regional Environment Programme and the Government of Western Samoa for supporting this position. We also thank all those who assisted with field work, especially David Butler, Julia Haska, Cedric Schuster and Pepper Trail. We are especially grateful to Jochen Gerber for extracting data for us from the Field Museum collections. Virginia Cowie labeled and databased much of our survey material in the Bishop Museum and Richard Pyle built the Samoan Snail Project website where data from the 1992-1994 survey can be accessed.

\section{References}

Alonso, A., Dallmeier, F., Granek, E., Raven, P., 2001. Biodiversity: Connecting with the Tapestry of Life. Smithsonian Institution/ Monitoring and Assessment of Biodiversity Program and President's Committee of Advisors on Science and Technology, Washington, DC.

Athens, J.S., Ward, J.V., 1993. Environmental change and prehistoric Polynesian settlement in Hawai'i. Asian Perspectives 32, 205-223.

Baker, H.B., 1938. Zonitid Snails from Pacific Islands Part 1. 1. Southern Genera of Microcystinae. Bernice P. Bishop Museum Bulletin 158, 1-102. pls. 1-20..

Baker, H.B., 1941. Zonitid snails from Pacific Islands Parts 3 and 4. 3. Genera other than Microcystinae. 4. Distribution and indexes. Bernice P. Bishop Museum Bulletin 166, 205-370. pls. 43-65.
Bauman, S., 1996. Diversity and decline of land snails on Rota. Mariana Islands American Malacological Bulletin 12, 13-27.

Blanvillain, C., Florent, C., Thenot, V., 2002. Land birds of Tuamotu Archipelago, Polynesia: relative abundance and changes during the 20th century with particular reference to the critically endangered Polynesian ground-dove (Gallicolumba erythroptera). Biological Conservation 103, 139-149.

Bouchet, P., Abdou, A., 2001. Recent extinct land snails (Euconulidae) from the Gambier Islands with remarkable apertural teeth. Pacific Science 55, 121-127.

Cameron, R.A.D., 1998. Dilemmas of rarity: biogeographical insights and conservation priorities for land Mollusca. Journal of Conchology Special Publication 2, 51-60.

Carlton, J.T., 1996. Biological invasions and cryptogenic species. Ecology 77, 1653-1655.

Civeyrel, L., Simberloff, D., 1996. A tale of two snails: is the cure worse than the disease? Biodiversity and Conservation 5, 12311252.

Clench, W.J., 1949. Cyclophoridae and Pupinidae of Caroline, Fijian, and Samoan Islands. Bernice P. Bishop Museum Bulletin 196, 1-52.

Cooke Jr., C.M., Kondo, Y., 1960. Revision of Tornatellinidae and Achatinellidae (Gastropoda, Pulmonata). Bernice P. Bishop Museum Bulletin 221, 1-303.

Coote, T., Loeve, E., Meyer, J.-Y., Clarke, D., 1999. Extant populations of endemic partulids on Tahiti, French Polynesia. Oryx 33, 215-222.

Cowie, R.H., 1992. Evolution and Extinction of Partulidae, endemic Pacific Island land snails. Philosophical Transactions of the Royal Society of London 335, 167-191.

Cowie, R.H., 1996a. Variation in species diversity and shell shape in Hawaiian land snails: in situ speciation and ecological relationships. Evolution 49 (6), 1191-1202.

Cowie, R.H., 1996b. Pacific island land snails: relationships, origins, and determinants of diversity. In: Keast, A., Miller, Scott, E. (Eds.), The Origin and Evolution of Pacific Island Biotas, New Guinea to Eastern Polynesia: Patterns and Processes. SPB Academic Publishing, Amsterdam, pp. 347-372.

Cowie, R.H., 1998. Catalog of the non-marine snails and slugs of the Samoan islands. Bishop Museum Bulletin in Zoology 3, i-viii 1-122.

Cowie, R.H., 2001a. Decline and homogenization of Pacific faunas: the land snails of American Samoa. Biological Conservation 99, 207-222.

Cowie, R.H., 2001b. Can snails ever be effective and safe biocontrol agents? International Journal of Pest Management 47, 23-40.

Cowie, R.H., 2002a. Invertebrate invasions on Pacific islands and the replacement of unique native faunas: a synthesis of the land and freshwater snails. Biological Invasions 3, 119-136.

Cowie, R.H., 2002b. Snail predator now in Samoa. Tentacle-Newsletter of the IUCN/SSC Mollusc Specialist Group 10, 18.

Cowie, R.H., Cook, R.P., 1999. The Distribution and Abundance of Land Snails in the National Park of American Samoa, with Particular Focus on Partulidae. Cooperative National Park Resources Studies Unit, University of Hawaii at Manoa, Technical Report 125.

Cowie, R.H., Cook, R.P., 2001. Extinction or survival: partulid tree snails in American Samoa. Biodiversity and Conservation 10, 143 159.

Cowie, R.H., Rundell, R.J., 2002. The land snails of a small tropical Pacific island, Aunu'u, American Samoa. Pacific Science 56, 143 147.

Cowie, R.H., Rundell, R.J., Mika, F., Setu, P. The endangered partulid tree snail Samoana thurstoni on Olosega and the land snail diversity of the Manu'a Islands, American Samoa. American Malacological Bulletin (in press).

Dahl, A.L., 1991. Island Directory. UNEP Regional Seas Directories and Bibliographies No. 35. United Nations Enviromment Programme, Nairobi. 
D’Antonio, C.M., Dudley, T.L., 1995. Biological invasions as agents of change on islands versus mainlands. In: Vitousek, P.M., Loope, L.L., Andersen, H. (Eds.), Islands-Biological Diversity and Ecosystem Function. Springer, Berlin, pp. 103-121.

Eldredge, L.G., 2000. Numbers of Hawaiian species. Supplement 5. Bishop Museum Occasional Papers 63, 3-8.

Emberton, K.C., Pearce, T.A., Randalana, R., 1996. Quantitatively sampling land-snail species richness in Madagascan rainforests. Malacologia 38, 203-212.

Girardi, E.-L., 1978. The Samoan land snail genus Ostodes (Mollusca: Prosobranchia: Poteriidae). The Veliger 20, 191-250.

Goodacre, S.L., Wade, C.M., 2001. Molecular evolutionary relationships between partulid land snails of the Pacific. Proceedings of the Royal Society of London B 268, 1-7.

Gould, S.J., 1991. Unenchanted evening. Natural History 9/ 91, 4-14.

Hadfield, M.G., 1986. Extinction in Hawaiian achatinelline snails. Malacologia 27, 67-81.

Hadfield, M.G., Miller, S.E., Carwile, A.H., 1993. The decimation of endemic Hawai'ian [sic] tree snails by alien predators. American Zoologist 33, 610-622.

Hopper, D.R., Smith, B.D., 1992. Status of tree snails (Gastropoda: Partulidae) on Guam, with a resurvey of sites studied by H. E. Crampton in 1920. Pacific Science 46, 77-85.

Hunt, T.L., Kirch, P.V., 1997. The historical ecology of Ofu Island, American Samoa, 3000 B.P. to the present. In: Kirch, P.V., Hunt, T.L. (Eds.), Historical Ecology in the Pacific Islands. Yale University Press, New Haven and London, pp. 105-123.

Johnson, M.S., Murray, J., Clarke, B., 1993. The ecological genetics and adaptive radiation of Partula on Moorea. Oxford Surveys in Evolutionary Biology 9, 167-238.

Keating, B.H., 1992. The geology of the Samoan Islands. In: Keating, B.H., Bolton, B.R. (Eds.), Geology and Offshore Mineral Resources of the Central Pacific Basin. Springer, New York, pp. 127-178.

Kirch, P.V., 1993. Non-marine molluscs from the To'aga site sediments and their implications for environmental change. Contributions of the University of California Archaeological Research Facility, Berkeley 51, 115-121.

Kurozumi, T., 1988. Species composition and abundance of land mollusks and factors affecting their extinction in the Ogasawara (Bonin) Islands. Ogasawara Research 14/15, 59-109.

Loope, L.L., 1998. Hawaii and the Pacific Islands. In: Mac, M.J., Opler, P.A., Haecker, C.E.P., Doran, P.D. (Eds.), Status and Trends of the Nation's Biological Resources. U.S. Department of the Interior, U.S. Geological Survey, Reston, pp. 747-774.

Murray, J., Murray, E., Johnson, M.S., Clarke, B., 1989. The extinction of Partula on Moorea. Pacific Science 42, 150-153.

Park, G., Hay, J., Whistler, W.A., Lovegrove, T., Ryan, P., 1992. The Ecological Survey of Western Samoa: The Conservation of Biological Diversity in the Coastal Lowlands of Western Samoa. Report by the New Zealand Department of Conservation for the Ministry of External Relations and Trade.
Paulay, G., 1994. Biodiversity on oceanic islands: its origin and extinction. American Zoologist 34, 134-144.

Pearsall, S.H., Whistler, W.A., 1991. Terrestrial Ecosystem Mapping for Western Samoa: summary, project report, and proposed national parks and reserves plan. Report prepared for the Government of Western Samoa. South Pacific Regional Environment Programme, Noumea, and East-West Center, Environment and Policy Institute, Honolulu.

Pearsall, S.H., Whistler, W.A., 1991. Ecosystem mapping for Western Samoa: technical report and appendices. Report prepared for the Government of Western Samoa. South Pacific Regional Environment Programme, Noumea, and East-West Center, Environment and Policy Institute.

Pilsbry, H.A., 1916-1918. Manual of Conchology. Second series: Pulmonata. Vol. XXIV Pupillidae (Gastrocoptinae). Academy of Natural Sciences, Philadelphia.

Sherley, G., 2000. Invasive Species in the Pacific: a Technical Review and Draft Regional Strategy. South Pacific Regional Environment Programme, Apia.

Simberloff, D., 2000. Extinction-proneness of island species-causes and management implications. Raffles Bulletin of Zoology 48, 1-9.

Simberloff, D. Ecological and economic impacts of alien species: a phenomenal global change. In: Claudi, R., Hendrickson, O., Ottens, H. (Eds.), Alien Invasive Species: a Threat to Canadian Biodiversity. Natural Resources Canada, Canadian Forest Service, Ottawa. (in press).

Solem, A., 1976. Endodontoid land snails from Pacific islands (Mollusca: Pulmonata: Sigmurethra). Part I. Family Endodontidae. Field Museum of Natural History, Chicago.

Solem, A., 1983. Endodontoid Land Snails from Pacific Islands (Mollusca: Pulmonata: Sigmurethra). Part II. Families Punctidae and Charopidae, Zoogeography. Field Museum of Natural History, Chicago.

Solem, A., 1990. How many Hawaiian land snail species are left? and what we can do for them. Bishop Museum Occasional Papers 30, $27-40$.

Taule'alo, T.I., 1993. Western Samoa: State of the Environment Report. South Pacific Regional Environment Programme, Apia.

Tomiyama, K., Kurozumi, T., 1992. Terrestrial mollusks and conservation of their environment in the Ogasawara Island. Regional Views 5, 39-81.

Ward-Booth, J.F., Dussart, G.B.J., 2001. Consistency in handsearching for terrestrial snails. Journal of Molluscan Studies 67, 502-506.

Whistler, W.A., 1992. Vegetation of Tonga and Samoa. Pacific Science 46, 159-178.

Whistler, W.A., 1993. The Cloud Forest of Samoa. In: Hamilton, L.S., Juvik, J.O., Scatena, F.N. (Eds.), Tropical Montane Cloud Forests. Proceedings of an International Symposium at San Juan, Puerto Rico 31 May-5 June 1993. East-West Center, Honolulu, pp. 231236. 